\title{
Toward Early Diagnosis of Lung Cancer
}

Ayman El-Baz ${ }^{1}$, Georgy Gimel'farb², Robert Falk ${ }^{3}$, Mohamed Abou El-Ghar ${ }^{4}$, Sabrina Rainey ${ }^{1}$, David Heredia ${ }^{1}$, and Teresa Shaffer ${ }^{1}$

1 Bioimaging Laboratory, Bioengineering Department, University of Louisville, Louisville, KY, USA

2 Department of Computer Science, University of Auckland, Auckland, New Zealand

3 Director, Medical Imaging Division, Jewish Hospital, Louisville, KY, USA

4 Urology and Nephrology Department, University of Mansoura, Mansoura, Egypt

\begin{abstract}
Our long term research goal is to develop a fully automated, image-based diagnostic system for early diagnosis of pulmonary nodules that may lead to lung cancer. In this paper, we focus on generating new probabilistic models for the estimated growth rate of the detected lung nodules from Low Dose Computed Tomography (LDCT). We propose a new methodology for 3D LDCT data registration which is non-rigid and involves two steps: (i) global target-to-prototype alignment of one scan to another using the learned prior appearance model followed by (ii) local alignment in order to correct for intricate relative deformations. Visual appearance of these chest images is described using a MarkovGibbs random field (MGRF) model with multiple pairwise interaction. An affine transformation that globally registers a target to a prototype is estimated by the gradient ascent-based maximization of a special Gibbs energy function. To handle local deformations, we displace each voxel of the target over evolving closed equi-spaced surfaces (iso-surfaces) to closely match the prototype. The evolution of the iso-surfaces is guided by a speed function in the directions that minimize distances between the corresponding voxel pairs on the iso-surfaces in both the data sets. Preliminary results show that the proposed accurate registration could lead to precise diagnosis and identification of the development of the detected pulmonary nodules.
\end{abstract}

\section{Introduction}

Because lung cancer is the most common cause of cancer deaths, fast and accurate analysis of pulmonary nodules is of major importance for medical computeraided diagnostic systems (CAD).

Previous work. Tracking the temporal nodule behavior is a challenging task because of changes in the patient's position at each data acquisition, as well as effects of heart beats and respiration. In order to accurately measure how the nodules are developing in time, all these motions should be compensated by registering LDCT data sets taken at different time. Many methods have been proposed for solving medical image registration problems (see e.g. [1]) and to exclude the lung motions (see 2]). Moreover, it has been reported that the 
computer-assisted volume measurement is more reliable for small pulmonary nodules than the measurement by human experts [3. Therefore, the remaining principal difficulty in monitoring and evaluating the nodule growth rate is automatic identification (or registration) of corresponding nodules in the follow-up scans. Registration of the two successive CT scans determines transformation of one image with respect to the other [4. Some examples of previous works on registration of CT lung images are overviewed below.

Most of them exploit corresponding local structural elements (features) in the images. For the follow-up of small nodules, Brown et al. [5] developed a patientspecific model with $81 \%$ success for 27 nodules. Ko et al. 6] used centroids of local structures to apply rigid and affine image registration with $96 \%$ success for 58 nodules of 10 patients. To account for non-rigid motions and deformations of the lung, Woods et al. 7] developed an objective function using an anisotropic smoothness constraint and a continuous mechanical model. Feature points required by this algorithm are detected and registered as explained in [8], and then the continuous mechanical model is used to interpolate the image displacement.

\section{Lung Motion Correction Models}

\subsection{Global Alignment}

Basic Notation. Let $\mathcal{Q}=\{0, \ldots, Q-1\} ; \mathbf{R}=[(x, y, z): x=0, \ldots, X-1 ; y=$ $0, \ldots, Y-1 ; z=0, \ldots, Z-1]$, and $\mathbf{R}_{\mathrm{p}} \subset \mathbf{R}$ be a finite set of scalar image signals (e.g. gray levels), a 3D arithmetic lattice supporting digital LDCT image data $g: \mathbf{R} \rightarrow \mathcal{Q}$, and an arbitrary-shaped part of the lattice occupied by the prototype, respectively. Let a finite set $\mathcal{N}=\left\{\left(\xi_{1}, \eta_{1}, \zeta_{1}\right), \ldots,\left(\xi_{n}, \eta_{n}, \zeta_{n}\right)\right\}$ of the $(x, y, z)$-coordinate offsets define neighboring voxels, or neighbors $\{((x+\xi, y+$ $\eta, z+\zeta),(x-\xi, y-\eta, z-\zeta)):(\xi, \eta, \zeta) \in \mathcal{N}\} \wedge \mathbf{R}_{\mathrm{p}}$ interacting with each voxel $(x, y, z) \in \mathbf{R}_{\mathrm{p}}$. The set $\mathcal{N}$ yields a $3 \mathrm{D}$ neighborhood graph on $\mathbf{R}_{\mathrm{p}}$ that specifies translation invariant pairwise interactions between the voxels with $n$ families $\mathcal{C}_{\xi, \eta, \zeta}$ of second-order cliques $c_{\xi, \eta, \zeta}(x, y, z)=((x, y, z),(x+\xi, y+\eta, z+\zeta))$. Interaction strengths are given by a vector $\mathbf{V}^{\top}=\left[\mathbf{V}_{\xi, \eta, \zeta}^{\top}:(\xi, \eta, \zeta) \in \mathcal{N}\right]$ of potentials $\mathbf{V}_{\xi, \eta, \zeta}^{\top}=\left[V_{\xi, \eta, \zeta}\left(q, q^{\prime}\right):\left(q, q^{\prime}\right) \in \mathcal{Q}^{2}\right]$ depending on signal co-occurrences; here $\mathrm{T}$ indicates transposition.

Data normalization. To account for possible monotone (order -preserving) changes of signals (e.g. due to different sensor characteristics), every LDCT data set is equalized using the cumulative empirical probability distribution of its signals.

Markov-Gibbs random field (MGRF) based appearance model. In a generic MGRF with multiple pairwise interaction, the Gibbs probability $P(g) \propto$ $\exp (E(g))$ of an object $g$ aligned with the prototype $g^{\circ}$ on $\mathbf{R}_{\mathrm{p}}$ is specified with the Gibbs energy $E(g)=\left|\mathbf{R}_{\mathbf{p}}\right| \mathbf{V}^{\top} \mathbf{F}(g)$ where $\mathbf{F}^{\top}(g)$ is the vector of scaled empirical probability distributions of signal co-occurrences over each clique family: $\mathbf{F}^{\top}(g)=\left[\rho_{\xi, \eta, \zeta} \mathbf{F}_{\xi, \eta, \zeta}^{\top}(g):(\xi, \eta, \zeta) \in \mathcal{N}\right]$ where $\rho_{\xi, \eta, \zeta}=\frac{\left|\mathcal{C}_{\xi, \eta, \zeta}\right|}{\left|\mathbf{R}_{p}\right|}$ is the relative size of the family and $\mathbf{F}_{\xi, \eta, \zeta}(g)=\left[f_{\xi, \eta, \zeta}\left(q, q^{\prime} \mid g\right):\left(q, q^{\prime}\right) \in \mathcal{Q}^{2}\right]^{\top}$; here, 
$f_{\xi, \eta, \zeta}\left(q, q^{\prime} \mid g\right)=\frac{\left|\mathcal{C}_{\xi, \eta, \zeta \zeta q, q^{\prime}}(g)\right|}{\left|\mathcal{C}_{\xi, \eta, \zeta}\right|}$ are empirical probabilities of signal co-occurrences, and $\mathcal{C}_{\xi, \eta, \zeta ; q, q^{\prime}}(g) \subseteq \mathcal{C}_{\xi, \eta, \zeta}$ is a subfamily of the cliques $c_{\xi, \eta, \zeta}(x, y, z)$ supporting the co-occurrence $\left(g_{x, y, z}=q, g_{x+\xi, y+\eta, z+\zeta}=q^{\prime}\right)$ in $g$. The co-occurrence distributions and the Gibbs energy for the object are determined over $\mathbf{R}_{\mathrm{p}}$, i.e. within the prototype boundary after an object is affinely aligned with the prototype. To account for the affine transformation, the initial image is resampled to the back-projected $\mathbf{R}_{\mathrm{p}}$ by interpolation.

Learning the potentials. The MLE of $\mathbf{V}$ is proportional in the first approximation to the scaled centered empirical co-occurrence distributions for the prototype:

$$
\mathbf{V}_{\xi, \eta, \zeta}=\lambda \rho_{\xi, \eta, \zeta}\left(\mathbf{F}_{\xi, \eta, \zeta}\left(g^{\circ}\right)-\frac{1}{Q^{2}} \mathbf{U}\right) ;(\xi, \eta, \zeta) \in \mathcal{N}
$$

where $\mathbf{U}$ is the vector with unit components. The common scaling factor $\lambda$ is also computed analytically; it is approximately equal to $Q^{2}$ if $Q \gg 1$ and $\rho_{\xi, \eta, \zeta} \approx 1$ for all $(\xi, \eta, \zeta) \in \mathcal{N}$. In our case it can be set to $\lambda=1$ because the registration uses only relative potential values and energies.

Learning the characteristic neighbors. To find the characteristic neighborhood set $\mathcal{N}$, the relative Gibbs energies $E_{\xi, \eta, \zeta}\left(g^{\circ}\right)=\rho_{\xi, \eta, \zeta} \mathbf{V}_{\xi, \eta, \zeta}^{\top} \mathbf{F}_{\xi, \eta, \zeta}\left(g^{\circ}\right)$ for the clique families, i.e. the scaled variances of the corresponding empirical cooccurrence distributions, are compared for a large number of possible candidates.

To automatically select the characteristic neighbors, we consider an empirical probability distribution of the energies as a mixture of a large "non-characteristic" low-energy component and a considerably smaller characteristic high-energy component: $P(E)=\pi P_{\mathrm{lo}}(E)+(1-\pi) P_{\mathrm{hi}}(E)$. Both the components $P_{\mathrm{lo}}(E), P_{\mathrm{hi}}(E)$ are of arbitrary shape and thus are approximated with linear combinations of positive and negative discrete Gaussians (EM-based algorithms introduced in [9] are used for both the approximation and the estimation of $\pi$ ). Example of the estimated characteristic neighbors is shown in Fig. 1]

Appearance-based registration. The desired affine transformation of an object $g$ corresponds to a local maximum of its relative energy $E\left(g_{\mathbf{a}}\right)=\mathbf{V}^{\top} \mathbf{F}\left(g_{\mathbf{a}}\right)$
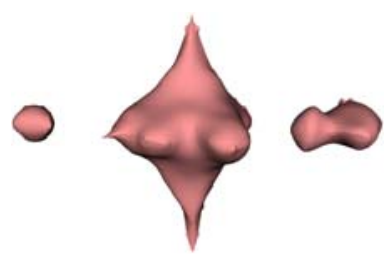

(a)
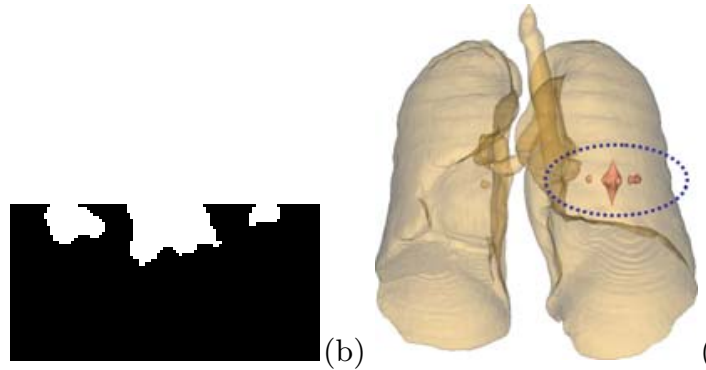

(c)

Fig. 1. The 3D neighborhood system (a) estimated for the lung tissues; its 2D cross section in the plane $\zeta=0$ (b; in white) and its superposition onto the lungs reconstructed from the LDCT images (c) 
under the learned appearance model $[\mathcal{N}, \mathbf{V}]$. Here, $g_{\mathbf{a}}$ is the part of the object image reduced to $\mathbf{R}_{\mathrm{p}}$ by the $3 \mathrm{D}$ affine transformation $\mathbf{a}=\left[a_{11}, \ldots, a_{34}\right]: x^{\prime}=$ $a_{11} x+a_{12} y+a_{13} z+a_{14} ; y^{\prime}=a_{21} x+a_{22} y+a_{23} z+a_{24} ; z^{\prime}=a_{31} x+a_{32} y+a_{33} z+a_{34}$. The initial transformation step is a pure translation with $a_{11}=a_{22}=a_{33}=1$; $a_{12}=a_{13}=a_{21}=a_{23}=a_{31}=a_{32}=0$, ensuring the most "energetic" overlap between the object and prototype. In other words, the chosen initial position $\left(a_{14}^{*}, a_{24}^{*}, a_{34}^{*}\right)$ maximizes the Gibbs energy. Then the gradient search for the local energy maximum closest to the initialization selects all the 12 parameters.

Figures 2 (c,d) show the results of the global alignment of two segmented lungs. It is clear from Fig. 2(d) that the global alignment is not perfect due to local deformation.

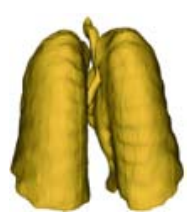

(a)

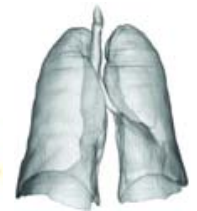

(b)

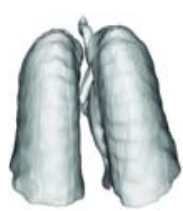

(c)

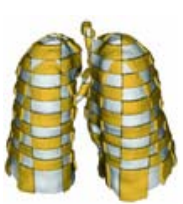

(d)

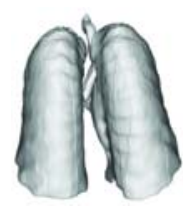

(e)

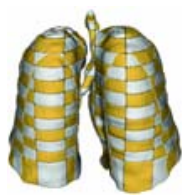

(f)

Fig. 2. 3D global and local registration: (a) reference data, (b) target data, (c) target data after $3 \mathrm{D}$ affine transformation, (d) checkerboard visualization to show the motion of lung tissues, (e) results of our non-rigid registration, and (f) checkerboard visualization to show the quality of the proposed local deformation model.

\subsection{Local Motion Model}

To handle local deformations, we propose to deform the object over evolving closed equi-spaced surfaces (distance iso-surfaces) so that it closely matches the prototype. The evolution is guided by an exponential speed function and intends to minimize distances between corresponding voxel pairs on the iso-surfaces in both the images. The normalized cross correlation of the Gibbs energy is used to find correspondences between the iso-surfaces.

Our approach involves the following steps. First, a distance map inside the object is generated using fast marching level sets [10. Secondly, the distance map is used to generate iso-surfaces (Fig. 3(b)). Note that the number of isosurfaces is not necessarily the same for both the images and depends on the accuracy and the speed required by the user. The third step consists in finding correspondences between the iso-surfaces using the normalized cross correlation of the Gibbs energy. Finally, the evolution process deforms the iso-surfaces in the first data set (the target image) to match the iso-surfaces in the second data set (the prototype).

The following notation is used below for defining the evolution equation:

$-\mathbf{b}_{\mathbf{g}_{1}}^{h}=\left[\mathbf{p}_{k}^{h}: k=1, \ldots, K\right]-K$ control points on a surface $h$ on the reference data such that $\mathbf{p}_{k}=\left(x_{k}, y_{k}, z_{k}\right)$ form a circularly connected chain of line segments $\left(\mathbf{p}_{1}, \mathbf{p}_{2}\right), \ldots,\left(\mathbf{p}_{K-1}, \mathbf{p}_{K}\right),\left(\mathbf{p}_{K}, \mathbf{p}_{1}\right)$; 
- $\mathbf{b}_{\mathbf{g}_{2}}^{\gamma}=\left[\mathbf{p}_{n}^{\gamma}: n=1, \ldots, N\right]-N$ control points on a surface $\gamma$ on the target data such that $\mathbf{p}_{n}=\left(x_{n}, y_{n}, z_{n}\right)$ form a circularly connected chain of line segments $\left(\mathbf{p}_{1}, \mathbf{p}_{2}\right), \ldots,\left(\mathbf{p}_{N-1}, \mathbf{p}_{N}\right),\left(\mathbf{p}_{N}, \mathbf{p}_{1}\right)$;

- $S\left(\mathbf{p}_{k}^{h}, \mathbf{p}_{n}^{\gamma}\right)$ - the Euclidean distance between a point on the surface $h$ in the image $\mathbf{g}_{1}$ and the corresponding point on the surface $\gamma$ in the image $\mathbf{g}_{2}$;

- $S\left(\mathbf{p}_{n}^{\gamma}, \mathbf{p}_{n}^{\gamma-1}\right)$ - the Euclidean distance between a point on the surface $\gamma$ in the image $\mathbf{g}_{1}$ and the nearest point on the surface $\gamma-1$ in $\mathbf{g}_{1}$, and

$-\nu($.$) - the propagation speed function.$

The evolution $\mathbf{b}_{\tau} \rightarrow \mathbf{b}_{\tau+1}$ of a deformable boundary $\mathbf{b}$ in discrete time, $\tau=$ $0,1, \ldots$, is specified by the system $\mathbf{p}_{n, \tau+1}^{\gamma}=\mathbf{p}_{n, \tau}^{\gamma}+\nu\left(\mathbf{p}_{n, \tau}^{\gamma}\right) \mathbf{u}_{n, \tau} ; n=1, \ldots, N$ of difference equations where $\nu\left(\mathbf{p}_{n, \tau}^{\gamma}\right)$ is a propagation speed function for the control point $\mathbf{p}_{\mathbf{n}, \tau}^{\gamma}$ and $\mathbf{u}_{n, \tau}$ is the unit vector along the ray between the two corresponding points. The propagation speed function

$$
\nu\left(\mathbf{p}_{n, \tau}^{\gamma}\right)=\min \left\{S\left(\mathbf{p}_{k}^{h}, \mathbf{p}_{n, \tau}^{\gamma}\right), S\left(\mathbf{p}_{n, \tau}^{\gamma}, \mathbf{p}_{n, \tau}^{\gamma-1}\right), S\left(\mathbf{p}_{n, \tau}^{\gamma}, \mathbf{p}_{n, \tau}^{\gamma+1}\right)\right\}
$$

satisfies the condition $\nu\left(\mathbf{p}_{n, \tau}^{\gamma}\right)=0$ if $S\left(\mathbf{p}_{k}^{h}, \mathbf{p}_{n, \tau}^{\gamma}\right)=0$ and prevents the current point from cross-passing the closest neighbor surfaces as shown in Fig. [3(a). The latter restriction is known as the smoothness constraint.

Again, the checkerboard visualization (Fig. 2(d)) of the data set in Fig. 2(a) and the aligned data set in Fig. 2(c) highlights the effect of the motion of lung tissues.
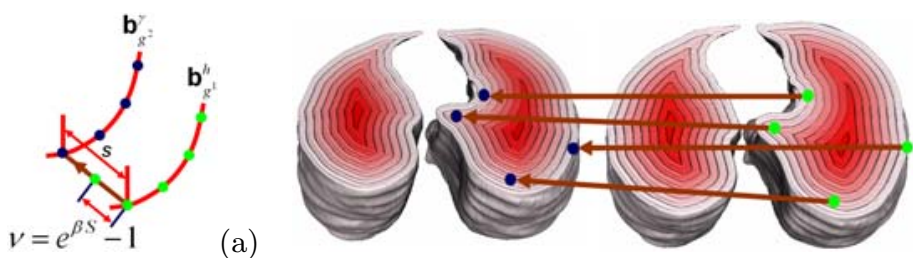

Fig. 3. (a) The proposed evolution scenario and (b) equi-spaced surfaces

\section{Experimental Results and Conclusions}

The proposed registration models were tested on the clinical datasets collected from 27 patients. Each patient has five LDCT scans, with the three months period between each two successive scans. This preliminary clinical database was collected by the LDCT scan protocol using a multidetector GE Light Speed Plus scanner with the following scanning parameters: slice thickness of $2.5 \mathrm{~mm}$ reconstructed every $1.5 \mathrm{~mm}$, scanning pitch 1.5, pitch $1 \mathrm{~mm}, 140 \mathrm{KV}, 100 \mathrm{MA}$, and F.O.V $36 \mathrm{~cm}$.

After the two volumes at different time instants are registered, the task is to find out if the nodules are growing or not. For this purpose, the lung nodules were segmented after registration using our previous approach [11. Once the nodules are segmented in the original and the registered image sequences, the 
Table 1. Growth rate statistics for 14 patients with malignant nodules and 13 patients with benign nodules $(p$ - statistical significance; $\mu$ - average rate, $\% ; \sigma$ - standard deviation, \%)

\begin{tabular}{|c|c|c|c|c|c|c|c|c|c|c|}
\hline \multirow{3}{*}{$\begin{array}{c}\text { Scanning } \\
\text { period }\end{array}$} & \multicolumn{5}{|c|}{ With the proposed registration } & \multicolumn{5}{|c|}{ Without the registration } \\
\hline & \multicolumn{2}{|c|}{ Malignant } & \multicolumn{2}{|c|}{ Benign } & \multirow[b]{2}{*}{$p$} & \multicolumn{2}{|c|}{ Malignan } & \multicolumn{2}{|c|}{ Benign } & \multirow[b]{2}{*}{$p$} \\
\hline & $\mu_{M}$ & $\sigma_{M}$ & $\mu_{B}$ & $\sigma_{B}$ & & $\mu_{M}$ & $\sigma_{M}$ & $\mu_{B}$ & $\sigma_{B}$ & \\
\hline 3 months & 22 & 16 & 0.9 & 0.7 & $10^{-4}$ & 5.6 & 4.8 & 2.8 & 1.9 & 0.1 \\
\hline 6 months & 49 & 20 & 2.9 & 2.3 & $10^{-}$ & 11 & 6.6 & 8.4 & 5.1 & 0.3 \\
\hline 9 months & 91 & 29 & 4.5 & 3.8 & $10^{-4}$ & 24 & $\overline{9.3}$ & 17 & 11 & 0.1 \\
\hline 12 months & 140 & 32 & 5.4 & 4.3 & $10^{-4}$ & 30 & 11 & 20 & 16 & 0.1 \\
\hline
\end{tabular}

Table 2. Statistical analysis for the growth rate of the detected lung nodules for fourteen patients who have malignant nodules and thirteen patients who have benign nodules using ImageChecker commercial CT CAD system

\begin{tabular}{|c|c|c|c|c|c|c|c|c|c|c|}
\hline \multirow{3}{*}{$\begin{array}{c}\text { Scanning } \\
\text { period }\end{array}$} & \multicolumn{5}{|c|}{ Diameter-based follow up } & \multicolumn{5}{|c|}{ Volume-based follow up } \\
\hline & \multicolumn{2}{|c|}{ Malignant } & \multicolumn{2}{|c|}{ Benign } & \multirow[b]{2}{*}{$p$} & \multicolumn{2}{|c|}{ Malignant } & \multicolumn{2}{|c|}{ Benign } & \multirow[b]{2}{*}{$p$} \\
\hline & $\mu_{M}$ & $\sigma_{M}$ & $\mu_{B}$ & $\sigma_{B}$ & & $\mu_{M}$ & $\sigma_{M}$ & $\mu_{B}$ & $\sigma_{B}$ & \\
\hline 3 months & 1.1 & 0.97 & 0.71 & 0.59 & 0.2229 & 6.15 & 3.91 & 3.67 & 2.73 & 0.0631 \\
\hline $6 \mathrm{~m}$ & 1.4 & 1.13 & 1.1 & 1.29 & 0.5254 & 11.7 & 4.37 & 9.27 & 4.17 & 0.152 \\
\hline 9 months & 1.8 & 2.77 & 1.6 & 2.51 & 0.8461 & 21.9 & 9.93 & 16.17 & 9.97 & 0.075 \\
\hline 12 months & 1.9 & 2.57 & 1.71 & 2.77 & 0.8548 & 31.3 & 12.3 & 22.21 & 12.7 & 0.070 \\
\hline
\end{tabular}

Table 3. Statistical analysis for the growth rate of the detected lung nodules for fourteen patients who have malignant nodules and thirteen patients who have benign nodules using the proposed approach in 12

\begin{tabular}{|c|c|c|c|c|c|}
\hline \multirow{2}{*}{$\begin{array}{c}\text { Scanning } \\
\text { period }\end{array}$} & \multicolumn{2}{|c|}{ Malignant } & \multicolumn{2}{|c|}{ Benign } & \multirow{2}{*}{} \\
\cline { 2 - 5 } & $\mu_{M}$ & $\sigma_{M}$ & $\mu_{B}$ & $\sigma_{B}$ & \\
\hline 3 months & 9.25 & 7.5 & 4.91 & 2.93 & 0.0624 \\
\hline 6 months & 16.1 & 11.97 & 9.95 & 6.91 & 0.1183 \\
\hline 9 months & 23.7 & 16.43 & 13.87 & 9.85 & 0.0737 \\
\hline 12 months & 45.57 & 34.87 & 25.57 & 15.77 & 0.0699 \\
\hline
\end{tabular}

volumes of the nodules are calculated using the $\Delta x, \Delta y$, and $\Delta z$ values from the scanner (in our case, $0.7,0.7$, and $2.5 \mathrm{~mm}$, respectively).

Our statistical analysis using the unpaired $t$-test shows that the difference between the average growth rate of malignant nodules and the average growth rate of benign nodules found with the proposed approach is statistically significant (as shown in Table 1). Also, Table 1 shows that no significant difference is found if the growth rate is measured without the data alignment step.

The advantages of using the proposed CAD system to estimate the growth rate of the detected lung nodules are highlighted by estimating the growth rate of the same detected lung nodules with ImageChecker commercial CT CAD system. This software provides two methods to monitor the detected lung nodules: 

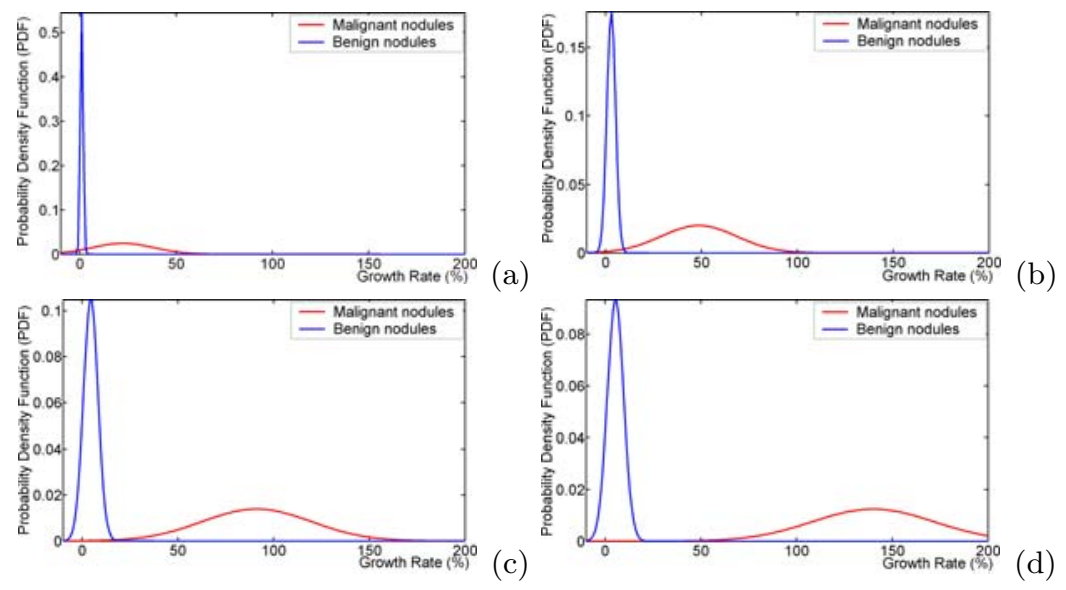

Fig. 4. Estimated probability density functions (probabilistic models) of the relative growth rates for 14 malignant and 13 benign nodules using our Linear Combination of Discrete Gaussian (LCDG) model [9]: (a) three months, (b) six months, (c) nine months, and (d) twelve months.

1) estimating the growth rate based on measuring the changes of the diameter of the largest cross section in the detected nodules and 2) estimating the growth rate based on measuring the volumetric changes of the detected nodules. The estimated growth rate using this CAD system is shown in Table 2. The main limitation of the ImageChecker CT CAD system is not considering the local deformation of the lung tissues from breathing and the heart beating. For this reason, the statistical analysis shown in Table 2 does not demonstrate a significant difference between the estimated growth rate of malignant lung nodules and the estimated growth rate of benign lung nodules, a difference which is detected by our proposed CAD system. The same limited ability to estimate the growth rate of the detected lung nodules exists in the most recent published work by Reeves et al. 12. The statistical analysis of the estimated growth rates of the same detected nodules using the proposed approach in [12] is shown in Table 3 . A traditional Bayes classifier based on the analysis of the growth rate of both benign and malignant nodules for 27 patients diagnosed 14 and 13 patients as malignant and benign, respectively. For simplicity, this classifier used a multivariate Gaussian model of the growth rate with the rates at 3, 6, 9, and 12 months as four discriminant features. The same patients were diagnosed by biopsy (the ground truth) showing that the classification was $100 \%$ correct. Therefore, the proposed image analysis techniques could be a promising supplement to the current technologies for diagnosing lung cancer.

We introduced a new approach for registering 3D spiral LDCT images that combines an initial affine global alignment of one scan (the target) to another scan (the reference) using the learned prior appearance model and subsequent local alignments that account for more intricate deformations. 


\section{References}

1. Maintz, J., Viergever, M.: A Survey of Medical Image Registration. Journal of Medical Image Analysis 2, 1-36 (1998)

2. Ko, J., Naidich, D.: Computer-Aided Diagnosis and the Evaluation of Lung Disease. Journal of Thoracic Imaging 19(3), 136-155 (2004)

3. Kostis, W., Yankelevitz, D., Reeves, A., Fluture, S., Henschke, C.: Small Pulmonary Nodules: Reproducibility of Three-Dimensional Volumetric Measurement and Estimation of Time to Follow-Up CT. Radiology 231(2), 446-452 (2004)

4. Horn, B.: Closed-Form Solution of Absolute Orientation using Unit Quaternions. Journal of the Optical Society of America B 4(4), 629-642 (1987)

5. Brown, M., McNitt-Gray, M., Mankovich, N., Goldin, J., Hiller, J., Wilson, L., Aberle, D.: Method for Segmenting Chest CT Image Data using an Anatomical Model: Preliminary Results. IEEE TMI 16(6), 828-839 (1997)

6. Ko, J., Betke, M.: Chest CT: Automated Nodule Detection and Assessment of Change over Time-Preliminary Experience. Radiology 218, 267-273 (2001)

7. Woods, K., Fan, L., Chen, C., Wang, Y.: Model Supported Image Registration and Warping for Change Detection in Computer-Aided Diagnosis. In: Applied Imagery Pattern Recognition (AIPR) Annual Workshops, Washington DC (2000)

8. Fan, L., Chen, C.: An Integrated Approach to 3D Warping and Registration from Lung Images. In: Proceedings of SPIE Conf. Developments in X-Ray Tomography II (July 1999)

9. El-Baz, A., Gimel'farb, G.: EM Based Approximation of Empirical Distributions with Linear Combinations of Discrete Gaussians. In: Proc. of IEEE International Conference on Image Processing (ICIP 2007), San Antonio, Texas, USA, September 16-19, vol. IV, pp. 373-376 (2007)

10. Sethian, J.: Fast Marching Level Set Method for Monotonically Advancing Fronts. Proc. National Academy of Sciences, USA 93, 1591-1595 (1996)

11. El-Baz, A., Farag, A., Gimel'farb, G., Falk, R., Abou El-Ghar, M., Eldiasty, T.: A Framework for Automatic Segmentation of Lung Nodules from Low Dose Chest CT Scans. In: Proc. IAPR Int. Conf. on Pattern Recognition (ICPR 2006), Hong Kong, August 20-24, vol. 3, pp. 611-614 (2006)

12. Reeves, A., Chan, A., Yankelevitz, D., Henschke, C., Kressler, B., Kostis, W.: On Measuring the Change in Size of Pulmonary Nodules. IEEE TMI 25(4), 435-450 (2006) 